\title{
Intrapulmonary Shunt Confirmed by Intracardiac Echocardiography in the Diagnosis of Hepatopulmonary Syndrome
}

\author{
Joseph E. Khabbaza, ${ }^{1}$ Richard A. Krasuski, ${ }^{2}$ and Adriano R. Tonelli ${ }^{1}$
}

$\mathrm{H}$ epatopulmonary syndrome (HPS) occurs in the setting of liver disease when oxygenation is impaired as a result of intrapulmonary vascular dilatation. The exact mechanism is not entirely clear, but is felt to be related to increases in pulmonary vasodilators, such as nitric oxide. ${ }^{1,2}$ Diagnosis requires the presence of liver disease, inadequate oxygenation, and confirmation of intrapulmonary shunting, generally by contrast-enhanced echocardiography. ${ }^{3,4}$ Occasionally, it can be difficult to decipher between intracardiac and intrapulmonary shunting. We report on a case of a male with cirrhosis who required the use of an intracardiac echocardiogram (ICE) for diagnosis of HPS.

\section{Case Report}

A 59-year-old male with cirrhosis secondary to alcohol use was referred to our pulmonary clinic for evaluation of hypoxemia and worsening dyspnea on exertion. Transthoracic echocardiogram (TTE) with saline contrast study suggested the presence of an interatrial septal defect. He had been started by a local physician on continuous oxygen and maintained at 2 $4 \mathrm{~L} / \mathrm{min}$. A repeat TTE with saline contrast showed normal right ventricle (RV) size and function, with an $\mathrm{RV}$ systolic pressure of $30 \mathrm{mmHg}$ and bubbles in the left atrium 5-6 beats after injection. His social history was pertinent for 40 years of heavy alcohol use, with his last drink 4 years earlier. His physical exam was only remarkable for a pulse oxygenation of $87 \%-93 \%$ on $2 \mathrm{~L} / \mathrm{min}$ of oxygen and significant lower extremity edema bilaterally. Heart examination revealed no murmurs or split-second sound.

A diffusing capacity corrected for hemoglobin was moderately reduced at $15.94 \mathrm{~mL} / \mathrm{min} / \mathrm{mmHg}(55 \%$ of predicted). Arterial blood gas standing and on room air revealed a $\mathrm{pH}$ of $7.45, \mathrm{pCO}_{2}$ of $31 \mathrm{mmHg}$, and $\mathrm{pO}_{2}$ of $61 \mathrm{mmHg}$ (measured alveolar-arterial gradient of $51.6 \mathrm{mmHg}$ ). A $100 \%$ oxygen shunt study showed a $\mathrm{pO}_{2}$ of $434 \mathrm{mmHg}$ with a calculated right-to-left shunt of $12.2 \%$. A transesophageal echocardiogram (TEE) could not be done because of esophageal varices, thus a right heart catheterization (RHC) was performed to better characterize whether there was a cardiac or a pulmonary shunt. RHC showed a pulmonary artery pressure of 36/22 (mean, $26 \mathrm{mmHg}$ ) with a pulmonary vascular resistance of 1.3 Wood units. ICE showed no heart shunt (Fig. 1A), but visualized bubbles coming into the left atrium from the pulmonary veins, confirming the presence of an intrapulmonary shunt and the diagnosis of HPS (Fig. 1B).

\section{Discussion}

HPS can generally be diagnosed with noninvasive testing. An elevated alveolar-arterial gradient occurs as the result of the dilatation of pulmonary vasculature, leading to shunt with ventilation-perfusion mismatch. ${ }^{5,6}$ Intrapulmonary shunting is most commonly demonstrated by contrast TTE when bubbles from

\footnotetext{
Abbreviations: HPS, hepatopulmonary syndrome; ICE, intracardiac echocardiogram; MAA, macroaggregated albumin; RHC, right heart catheterization; RV, right ventricle; TEE, transesophageal echocardiogram; TTE, transthoracic echocardiogram.

From the ${ }^{1}$ Department of Pulmonary and Critical Care, Respiratory Institute, Cleveland Clinic, Cleveland, OH; ${ }^{2}$ Department of Cardiovascular Medicine, Heart and Vascular Institute, Cleveland Clinic, Cleveland, $\mathrm{OH}$.

Received March 17, 2013; accepted April 11, 2013.

This publication was made possible by CTSA KL2 (grant no.: RR024990; to A.R.T.) from the National Center for Research Resources, a component of the National Institutes of Health (NIH), and the NIH Roadmap for Medical Research.

Address reprint requests to: Adriano Tonelli, M.D., Department of Pulmonary and Critical Care, Respiratory Institute, Cleveland Clinic, 9500 Euclid Avenue A-90, Cleveland, OH 44195. E-mail: tonella@ccf.org; fax: 216-445-6024.

Copyright $(2013$ by the American Association for the Study of Liver Diseases.

View this article online at wileyonlinelibrary.com.

DOI 10.1002/hep.26482

Potential conflict of interest: Dr. Krasuski consults and is on the speakers' bureau for Actelion. He consults for Gilead and advises Ventripoint.
} 

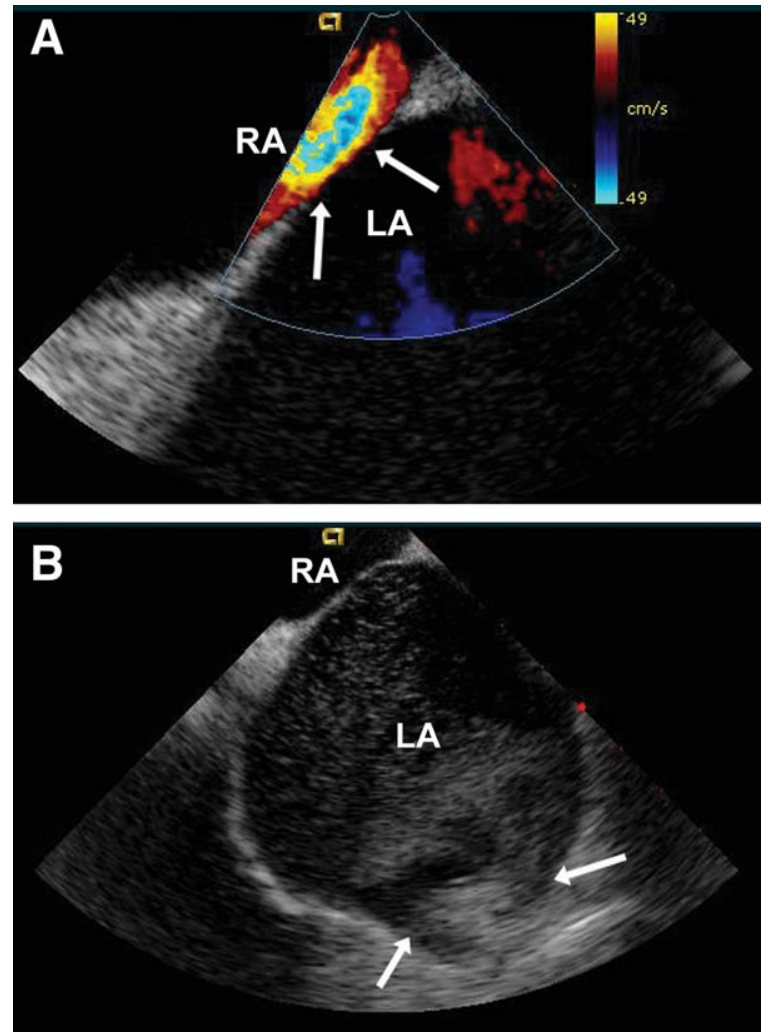

Fig. 1. (A) Doppler ICE showing no interatrial septal defect. Arrows point to the interatrial septum. LA, left atrium; RA, right atrium. (B) Two-dimensional ICE revealing bubbles in the left atrium after injection of agitated saline in the pulmonary artery. Arrows point to the left pulmonary veins (upper and lower). LA, left atrium; RA, right atrium.

agitated saline are visualized in the left atrium within 3-6 beats after being noted in the right side of the heart. ${ }^{1-3,5,7}$ Bubbles are not normally observed in the absence of vascular dilatation because lung capillaries act as filters. Another useful test for diagnosing HPS is the macroaggregated albumin (MAA) lung perfusion $\operatorname{scan}^{8}$; however, it cannot decipher the location of the shunt. $^{5}$

The presence of an intracardiac shunt, such as a patent foramen ovale or atrial septal defect, can affect the results of both contrast TTE and MAA lung perfusion scan. ${ }^{5}$ When an intracardiac shunt is present, bubbles cross through the atrial defect into the left atrium quickly, within 3 heart beats, ${ }^{3,9}$ as opposed to up to 6 beats with the intrapulmonary shunt. However, in the setting of large pulmonary shunts, bubbles may reach the left atrium much quicker, making the confirmation less certain. ${ }^{9}$ In this scenario, contrast TEE is superior to TTE because it directly visualizes the bubbles as they exit the pulmonary veins. ${ }^{5,9,10}$

The patient described had a contrast TTE that suggested both intracardiac and intrapulmonary shunting. A contrast TEE could not be performed because of the presence of esophageal varices; therefore, we performed an RHC with agitated saline directly administered into the pulmonary artery. Bubbles were visualized by ICE soon after within the left atrium, confirming the diagnosis of HPS. In addition, ICE ruled out the presence of a cardiac shunt. To our knowledge, this is the first case of HPS diagnosed by direct injection of bubbles into the pulmonary artery with the use of ICE. This minimally invasive approach may be useful in deciphering between intrapulmonary and -cardiac shunts in those with contraindications to TEE.

\section{References}

1. Rodriguez-Roisin R, Krowka MJ. Hepatopulmonary syndrome-a liver-induced lung vascular disorder. N Engl J Med 2008;358:23782387.

2. Machicao VI, Fallon MB. Hepatopulmonary syndrome. Semin Respir Crit Care Med 2012;33:11-16.

3. Pacca R, Maddukuri P, Pandian NG, Kuvin JT. Echocardiographic detection of intrapulmonary shunting in a patient with hepatopulmonary syndrome: case report and review of the literature. Echocardiography 2006;23:56-59.

4. Abrams GA, Jaffe CC, Hoffer PB, Binder HJ, Fallon MB. Diagnostic utility of contrast echocardiography and lung perfusion scan in patients with hepatopulmonary syndrome. Gastroenterology 1995;109:12831288 .

5. McAdams HP, Erasmus J, Crockett R, Mitchell J, Godwin JD, McDermott VG. The hepatopulmonary syndrome: radiologic findings in 10 patients. Am J Roentgenol 1996;166:1379-1385.

6. Lee KN, Lee HJ, Shin WW, Webb WR. Hypoxemia and liver cirrhosis (hepatopulmonary syndrome) in eight patients: comparison of the central and peripheral pulmonary vasculature. Radiology 1999;211:549553.

7. Schraufnagel DE, Kay JM. Structural and pathologic changes in the lung vasculature in chronic liver disease. Clin Chest Med 1996;17:1-15.

8. Abrams HA, Nanda NC, Dubovsky EV, Krowka MJ, Fallon MB. Use of macroaggregated albumin lung perfusion can to diagnose hepatopulmonary syndrome: a new approach. Gastroenterology 1998;114:305310.

9. Attaran RR, Ata I, Kudithipudi V, Foster L, Sorrell VL. Protocol for optimal detection and exclusion of a patent foramen ovale using transthoracic echocardiography with agitated saline microbubbles. Echocardiography 2006;23:616-622.

10. Vedrinne JM, Duperret S, Bizollon T, Magnin C, Motin J, Trepo C, Ducerf C. Comparison of transesophageal and transthoracic contrast echocardiography for detection of an intrapulmonary shunt in liver disease. Chest 1997;111:1236-1240. 